\title{
RECORRIDOS DE FORMACIÓN EN PSICOLOGÍA CLÍNICA Y DE LA SALUD EN ESPAÑA
}

\section{TRAINING CAREER IN CLINICAL AND HEALTH PSYCHOLOGY IN SPAIN}

\author{
Dr. Adolfo Jarne Esparcia \\ Profesor Titular de Psicopatología de la Universitat de Barcelona (UB). España \\ Miembro del Consejo Nacional de Acreditaciones en Psicología del CGPE \\ ORCID: https://orcid.org/0000-0002-4666-0704
}

\author{
Dra. Alba Pérez-González \\ Profesora Lectora de la Universitat Oberta de Catalunya (UOC). España \\ ORCID: https://orcid.org/0000-0003-2151-2198
}

Cómo referenciar este artículo/How to reference this article:

Jarne Esparcia, A. y Pérez-González, A. (2020). Recorridos de formación en psicología clínica y de la salud en España. Revista de Psicoterapia, 31(116), 77-85. https://doi.org/0.33898/rdp.v31i116.407

\begin{abstract}
Resumen
En este artículo se explica cuál es la situación legal actual de la práctica de la Psicología Clínica y Sanitaria en España y cuáles son los recorridos de formación en esta área en consonancia o no con la regulación legal. Se proponen seis recorridos posibles y se analizan las ventajas e inconvenientes de cada uno de ellos, así como su duración. Finalmente se mencionan los puntos de interés, a juicio de los autores, en el desarrollo de esta disciplina profesional y que en esencia son; (1) la incorporación al programa PIRy por lo tanto al acceso al título de Psicólogo Especialista en Psicología Clínica, de nuevas especialidades sanitarias psicológicas, (2) la posibilidad de incorporar a los Psicólogos Generales Sanitarios en la red pública y, por ende la cuestión de la relación entre los PEPC y los PGS, (3) por último, la necesidad de regulación legal de aquellos circuitos formativos que hoy en día se encuentran en una situación alegal, como podrían ser la neuropsicología o la psicoterapia.
\end{abstract}

Palabras clave: Formación, Psicología clínica y de la salud, Regulación, Profesión, Especialidades.

\begin{abstract}
This paper displays the current legal situation of the Clinical and Health psychological practice in Spain and explores if the training career in this domain is consistent or not with the Spanish legal regulation. Six possible training options with their respective lengths are proposed and the advantages and disadvantages of each are analyzed. Finally, current challenges are mentioned, according to authors 'opinions, in the development of this professional discipline. These challenges include (1) the inclusion of new psychological health specialties into the PIR program and therefore the access to the title of Specialist Psychologist in Clinical Psychology,(2) the incorporation of the General Health Psychologists in the public health system and, closely related, the relationship between the PEPC and the $P G S$, (3) finally, the need for legal regulation of those training options that are currently in a lackoflegal status situation, such as neuropsychology or psychotherapy.

Keywords: Training, Clinical and Health Psychology, Regulation, Profession, Specialties.
\end{abstract}




\section{Las especialidades psicológicas. Las especialidades en el área de la salud}

Es sabido que la práctica legal de la psicología clínica y de la salud en España (PCS en adelante), se articula alrededor de dos títulos con valor oficial ante las estructuras del estado; el del Psicólogo Especialista en Psicología Clínica y el del Psicólogo/a General Sanitario. En la práctica y por aplicación de la sentencia 372/2016 de la sección 6 a de la sala de lo Contencioso Administrativo, de la Audiencia Nacional de fecha 07/10/2016 ambos tienes iguales competencias y realizan iguales funciones sólo que las plazas de psicólogo/a especialista en psicología clínica en la red de asistencia pública y/o concertada con la administración, han de ser cubiertas por especialista en psicología clínica, quedando por lo tanto el título de psicólogo/a general sanitario circunscrito a lo que se denomina la práctica privada.

Es ahora el momento de diferenciar el sentido distinto que la palabra "especialista” adquiere en este contexto. En el sentido utilizado en el párrafo anterior significa un título oficial adquirido a través del programa de formación de especialista de la red pública sanitaria del estado español. Pero en un sentido más amplio viene a significar la adquisición de competencias y habilidades en un área determinada de un campo, en este caso de la práctica profesional de la psicología. Es en este segundo sentido en el que queremos continuar desarrollando nuestros argumentos.

No pretendemos justificar aquí, no creemos que haya que hacerlo, que todos los psicólogos pertenecemos, nos enorgullecemos de pertenecer, al tronco común de la "psicología” y, que la práctica clínica constituye una especialidad de ésta. De hecho no es este el lugar tampoco para explicar el proceso de ordenación del oficio del psicólogo y, como está resultando en sus especialidades más o menos reconocidas de forma fáctica. Si sirviera como referencia, el Consejo General de la Psicología de España tiene en este momento el siguiente programa de "acreditaciones”; Neuropsicología Clínica, Psicooncología y Cuidados Paliativos, Psicología de Emergencias, Psicología de la Educación, Psicología de la Intervención Social, Psicología del Deporte y Psicología Aeronáutica, con las siguientes propuestas de incorporación; Psicología Jurídica y Forense y, Psicología del Tráfico y la Seguridad. A ello hay que añadir la adhesión a la acreditación en Psicoterapia de la EFPA (Federación Europea de Asociaciones de Psicología).

Hay que resaltar con firmeza que la PCS posiblemente sea el área más prevalente de ellas, no solo por el lugar que ocupa en la misma historia de la psicología, sino también por su relevancia social y el porcentaje de psicólogos/as que se identifican con ella.

Lo curioso es que la PCS constituye una especialidad tan engrandecida dentro del mundo de la psicología que de hecho se configura como un área, que contiene en sí misma otras especialidades que se comportan de una forma muy autónoma unas de otras, aun aceptando su pertenencia al mismos tronco común; la PCS. Este fenómeno se produce porque al contrario que en la medicina, donde existe un área común, la salud, en psicología; hay psicólogos/as especializados en ámbito sanitario, educación, forense, emergencias, organizaciones sociales, intervención 
social, deporte... En sentido amplio todo ello es salud, pero en un sentido real, administrativo y organizacional, no.

El resultado es que probablemente nos es más fácil pensar en la PCS como una gran área de la psicología que no como una especialidad y esta gran área, lógicamente tiene especialidades.

Las especialidades en esta área vienen determinadas en nuestra opinión por dos vectores; la población de aplicación y el marco de actuación. Según la primera se constituyen la salud mental que tiene tendencia a "monopolizar" la denominación "psicología clínica”, la salud mental infanto-juvenil, la neuropsicología, la psicoterapia, la psicooncología y cuidados paliativos y, quizás una genérica de psicología de la salud. Según el marco, la especialidad en el sentido amplio, no oficial, se establece en intervención cognitivo-conductual, sistémica, humanista, integradora y los circuitos de formación en dinámica y psicoanálisis que libran su propia batalla muy al margen de los demás. Nosotros no somos partidarios de convertir este segundo vector en especialidades reconocidas por título oficial, pero si aceptar que constituyen circuitos de formación especializada.

En el resto del texto por lo tanto nos referiremos a la formación y los recorridos en PCS en el sentido amplio defendido en los párrafos anteriores y no en su sentido legal a menos que se haga mención explícita a ello.

\section{Niveles de formación}

Los niveles de formación en PCS en España son muy claros y fáciles de identificar. El primer nivel lo constituye el grado, que marca el título oficial de graduado en Psicología y que es imprescindible para pasar a los niveles siguientes. A pesar de que en su momento se pensó que se le podía dotar de algunas competencias profesionales sencillas concretas en PCS, (Libro Blanco de Psicología, ANECA, 2005) , este modelo de compartimentación de competencias no encaja en la realidad de nuestra práctica clínica, por lo que, en nuestra opinión, ese intento debe darse por muerto. Simplemente el título de grado en Psicología no faculta ni legal ni competencialmente para la práctica de ningún tipo y a ningún nivel de la PCS.

El segundo nivel lo constituye el máster; aquí hay que diferenciar entre dos másteres; el Máster Universitario en Psicología General Sanitaria (MUPGS a partir de ahora) y los títulos propios de cada universidad/institución. El primero marca, tal como hemos dicho, uno de los dos títulos oficiales en España, que faculta legalmente para la práctica de la PCS. Es más difícil responder a la pregunta de qué paquete de competencias exactamente dota, ya que en su desarrollo académico real, a pesar de existir directrices generales con número de créditos fijos asignados, cada universidad lo ha estructurado de acuerdo a su idiosincrasia o mejor dicho posiblemente a la idiosincrasia del grupo de dentro de los departamentos de Psicología que lo organiza en su momento. Es decir, en nuestra opinión, es imposible saber un titulado general sanitario en que competencias reales ha sido formado más allá de una mención vaga al diagnóstico, evaluación, intervención y 
tratamiento en enfermedades, trastornos y problemas clínicos mentales y de la salud relacionada con la psicología. No sabremos si tiene formación en psicoterapia o no, con que orientación; si la tiene en rehabilitación, si estudió neuropsicología o terapia sistémica; si vio trastornos de la alimentación y/o de la sexualidad, con que poblaciones realizó las prácticas. Para ello hay que preguntarle en que programa y en qué universidad. Por ello, con un gran sentido práctico, seguimos nosotros defendiendo que se conteste a esta pregunta con la respuesta de "competencias en la práctica de la PCS en el contexto privado".

Los títulos propios constituyen in sensu stricto la auténtica expertez ya que forman competencias en neuropsicología, terapia sistémica, rehabilitación cognitiva, psicoterapia integrativa, etc. No constituyen título oficial, luego no son necesarios desde la perspectiva legal para la práctica de la PCS en nuestro país. Por el contrario, constituyen la garantía de formación y competencia en esa área de especialización. Es importante esta idea de que, en nuestra opinión, no hay contradicción entre la formación en PGS y la especialización a través de títulos propios (másteres específicos). De hecho, hay una cierta estructura jerárquica lógica que coloca primera la formación en PGS y la especialización a través de título propio... a quien lo desee.

Queda finalmente la formación a través del programa de Psicólogo Especialista en Psicología Clínica (PEPC a partir de ahora). Su estructura y prestigio son sobradamente conocidos y sus problemas también. Probablemente el más importante es su escasa oferta de plazas. A pesar de que va en aumento progresivo, no ha alcanzado la cantidad de 200 anuales (168 en la del 2019) ¡para una población de 47 millones de habitantes! Una segunda cuestión es que queda en la práctica circunscrito a salud mental con incursiones en otras áreas, pero sin la profundidad suficiente como para considerarse una formación de especialista. Ello sin ahondar en la problemática de la atención psicológica en atención primaria y otras cuestiones que no se pueden abordar aquí. Desconocemos si el número de especialistas anuales son suficientes para afrontar las necesidades de la red pública, aunque intuimos que no, de hecho el último informe publicado por el Defensor del Pueblo (2020) recoge que hay 6 psicólogos clínicos por cada 100.000 habitantes, estos son 3 veces menos que la media europea que se sitúa en 18 , y por supuesto sin entrar a valorar la demanda de atención privada tal como es frecuente en España. No está claro si la solución del PGS fue acertada o no o, lo fue en su momento pero necesitará una revisión más adelante, pero ahí está.

¿A qué nivel se coloca la formación PEPC?, púes se quiera o no se quiera, a nivel legal es al mismo que el PGS; ambos títulos oficiales con ámbitos de actuación restringido para el PGS. A nivel competencial real tal como hemos dicho, el PEPC lo está en psicología clínica de lo que tradicionalmente se ha vinculado a salud mental y no en otras áreas de especialización como neuropsicología o psicoterapia o terapia familiar, por más que en la formación durante los cuatro años el residente recibiera algunos contenidos y/o hiciera prácticas durante algún tiempo en servicios vinculados. 
En la línea de este trabajo si algún día la red pública ofertara plazas de esta naturaleza (neuropsicología, psicoterapia, etc..) tendría que plantearse cómo lo hace; incluirlas específicamente en el sistema PIR diferenciándolas de las plazas en PEPC, en la línea de PENC (Psicólogo Especialista en Neuropsicología Clínica), en el mismo paquete que el PEPC con subespecialidades o en alguna forma de colaboración y reconocimiento de formación externa, ...

\section{Recorridos de formación en Psicología Clínica y de la Salud en España}

A nuestro saber existen las siguientes posibilidades de formación, expuestas por lo que sería nivel de preferencia (tabla 1 y figura 1).

Tabla 1. Itinerarios formativos posibles en Psicología Clínica y de la Salud.

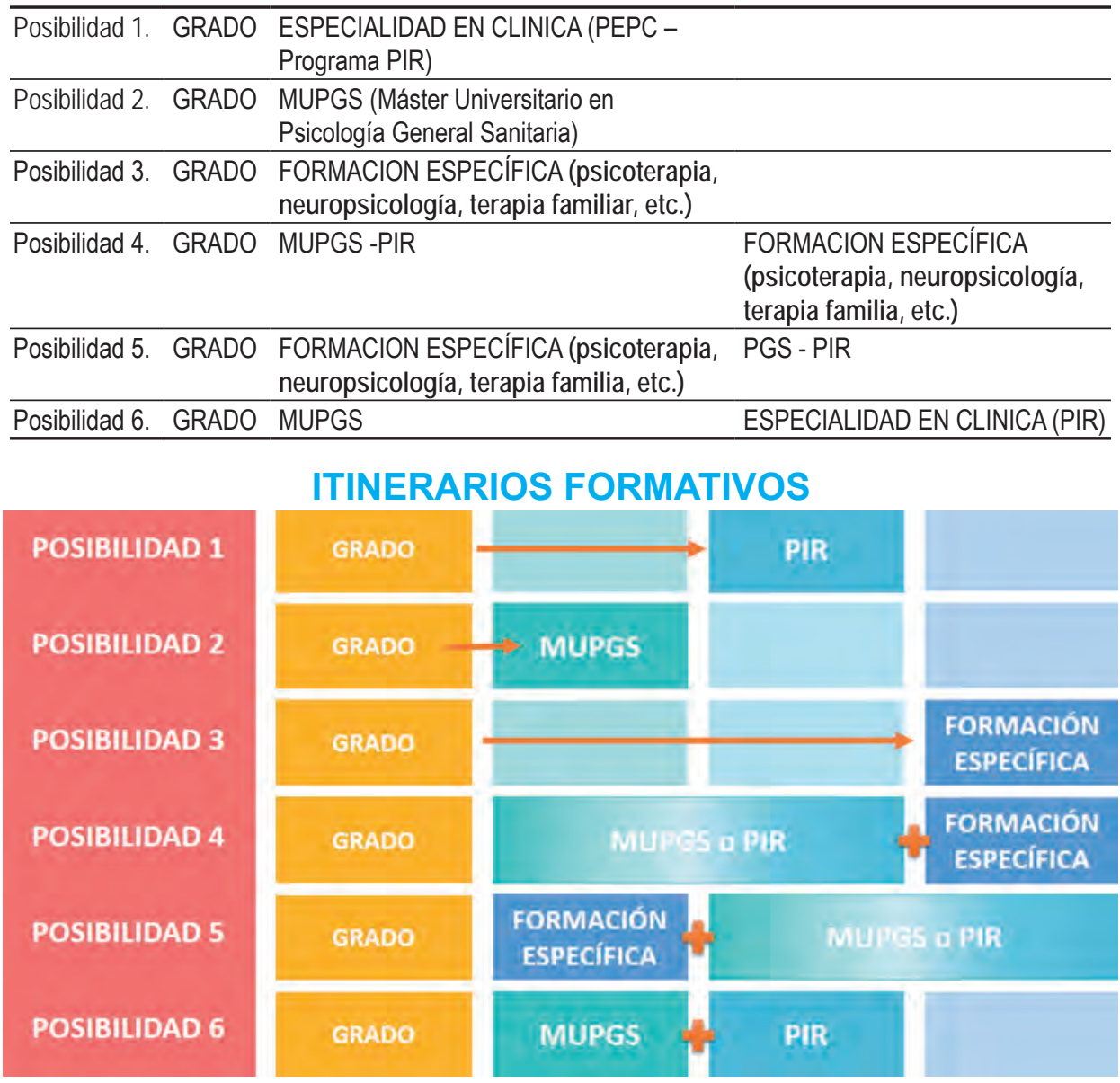

Figura 1. Itinerarios formativos posibles en Psicología Clínica y de Salud.

En la posibilidad 1 (Grado - PIR), tras el grado se accede al programa PIR y se obtiene el título de Psicólogo Especialista en Psicología Clínica. Tiene la 
ventaja de que se accede al título oficial sin restricciones, con la posibilidad por lo tanto de acceder al trabajo tanto en la red pública como en dispositivos privados. La formación es de alta calidad y esta remunerada mientras se realiza. Dada la tasa actual de reposición de PEPC (por jubilación de la primera hornada de habilitados en Psicología Clínica), es de prever la ausencia de paro en muchos años.

Los inconvenientes más importantes, además de la mencionada parquedad de plazas con la consiguiente dificultad para acceder a ellas, son la tendencia de formación en los trastornos mentales severos en contexto de red pública con menor incidencia en los trastornos emocionales frecuentes y en las técnicas de abordaje de tipo psicoterapia interpersonal y otras especialidades clínicas como neuropsicología o psicooncología.

En total la formación durará 8 años (cuatro de grado y cuatro PIR).

En la posibilidad 2 (Grado - MUPGS). Tras el grado se accede a un máster universitario en psicología general sanitaria (MUPGS) en cualquier universidad española con máster autorizado por las agencias de calidad, en cualquiera de las modalidades establecidas (presencial, semipresencial y a distancia). Ello no resulta difícil ya que la oferta es muy amplia. Las ventajas son que se obtiene un título oficial en las condiciones ya mencionadas. La calidad de la formación puede ser muy variable y, a nuestro saber, no existen datos respecto a este tema en este momento.

Los inconvenientes son los derivados de la falta de información sobre calidad de los diferentes programas tanto en la formación en contenidos como en las prácticas. También está el hecho de que algunos programas no ofertan formación, en nuestra opinión equivocadamente, en trastornos mentales severos, al considerar que la mayoría de estos pacientes son atendidos en red pública y por lo tanto fuera de las competencias legales del título.

Quizás el mayor inconveniente es estructural y es que es difícil definir en que constituye esta especialización más allá del grado. Conceptualmente es una formación general para una práctica general, sin que se haya llegado a definir exactamente qué significa ello en clínica psicológica.

En total la formación durará 6 años (cuatro de grado y dos en MUPGS).

En la posibilidad 3 (Grado - Formación Específica). Tras el grado, el psicóloga/a decide que quiere acceder directamente a una formación específica aunque no tenga añadido un título oficial; el caso paradigmático es Neuropsicología. La formación en neuropsicología clínica es tan compleja y exige tanta dedicación y tiempo que no puede ser contemplada y de hecho no lo es, ni en la formación del PEPC y en la del PGS. Sin embargo, no existe en España el título oficial de neuropsicólogo/a. Ello puede ser extensible a otras áreas de la salud como psicooncología o la formación en psicoterapia.

Las ventajas son obvias, se gana tiempo en el acceso a una formación de alta expertez, cuya calidad depende lógicamente del programa de formación, que está constituida por títulos propios de las universidades (sean oficiales o no de la misma universidad, lo que no significa que son oficiales para el estado; lo que lleva a fre- 
cuente confusión entre los usuarios. En nuestra opinión, las universidades deberían evitar la denominación "oficial”).

Los inconvenientes también son obvios. Tras la finalización de la formación, el psicólogo/a no tiene ningún título legal específico para la práctica de la PCS. Tiene una presumiblemente alta competencia técnica, pero no título legal.

¿Se puede practicar de esta forma? La respuesta es compleja, pero la resumiremos diciendo que no y sí. No se puede practicar las competencias adquiridas bajo el paraguas de Psicología Clínica, ya que la persona no tiene el título de PEPC, ni Psicología Sanitaria, ya que tampoco tiene el de PGS. Pero lo cierto es que en este momento en España, no está regulada ni la práctica de la Psicoterapia, que legalmente queda abierta a cualquier persona con titulación de grado en salud, ni la neuropsicología, por ejemplo; por lo que mientras una persona evite las denominaciones “clínicas” y/o "sanitarias”, existe la posibilidad de que pueda ejercer las competencias adquiridas.

En resumen, es un caso típico de discordancia entre la legalidad y la realidad y, queda la duda de la posibilidad de que un psicólogo/a practique la psicoterapia o la neuropsicología en un contexto privado si se circunscribe a dejarlo claro así “lo que hago es psicoterapia y neuropsicología, no PCS”. No creemos que esté en una situación ilegal sino alegal que es diferente.

Ello tiene su importancia: por ejemplo, los hospitales de la red pública tienen la necesidad de contratar neuropsicólogos/as. No pueden contratar PGS con formación posterior o previa en neuropsicología, porque éstos no pueden ejercer en ámbito de asistencia pública, y puede ser muy difícil encontrar PEPC con buena formación en neuropsicología. Se encuentran en un callejón sin salida que cada comunidad con competencias en sanidad lo soluciona a su manera. La solución catalana para la neuropsicología nos parece de la más elegante.

En total la formación durará 6 años (cuatro de grado y mínimo dos en máster especializado dependiendo de los años que recoja cada máster).

Posibilidad 4 (Grado - MUPGS/PIR - Formación Específica). Esta posibilidad es una de las más completas en nuestra opinión. Las ventajas provienen de que se adquiere una buena formación especializada que prepara a una práctica con alto nivel, con título legal, en el ámbito privado, lo que también dará paso a la consecución de las acreditaciones colegiales, que reconocen al profesional como de “práctica excelente”.

Los inconvenientes son la larga duración de la formación y su subsiguiente coste económico y vital.

En total la formación durará mínimo 8 años (cuatro de grado, entre dos y cuatro de MUPGS o PIR y otros dos en máster específico).Ello en sí mismo, no es una duración excesiva. En la comunidad europea se calcula que la formación de un clínico competente (de cualquier rama) está entre los 5 y los 12 años, con una media de 7.75 años, prácticamente 8 (Comisión Europea, 2016). La formación de un médico especialista en España es de 9 años (cinco de grado y cuatro de MIR). 
No obstante, la objeción de la carga que ello implica para las finanzas y el ciclo vital de una persona sigue estando sobre la mesa.

Posibilidad 5 (Grado- Formación Específica-MUPGS/PIR). Esta es una posibilidad muy poco utilizada, pero que en nuestra opinión debe ser tenida en cuenta; combina las posibilidades 2, 3 y 4 solo que con el control del tempus por parte del psicólogo/a. En esencia consiste en acogerse a la posibilidad 3; es decir, realizar un master altamente especializado tras el grado y cuando el profesional sienta la necesidad o bien por parte de la presión del mercado o por motivación interna/ personal realizar el MUPGS (lo más usual) o el programa PIR (lo menos frecuente). El valor añadido es que el profesional prioriza su formación de expertez sobre la titulación legal y busca ésta cuando siente la necesidad de esta. Naturalmente, ello pone sobre la mesa, no sobre el papel, la cuestión de cuán es realmente necesaria la titulación legal para la práctica privada de la psicología clínica/sanitaria y análogos en España, pero es algo de lo que no disponemos de datos para responder.

Posibilidad 6 (Grado - MUPGS - PIR). Desconocemos si alguna persona ha realizado el trayecto 6; es decir tras realizar el MUPGS, realiza el programa PIR y dispone por lo tanto de los dos títulos oficiales. Intuimos que si existe, son pocas personas.

Esta posibilidad se inscribe más en el conjunto de propuestas de la posible reordenación de la titulación legal para la práctica de la PCS y por lo tanto de rebote de los estudios universitarios relacionados. Una cantidad considerable de personas provenientes tanto de los colectivos de PEPC, como de los Colegios Profesionales de la Psicología, piensan que debería ser la trayectoria oficial y que por lo tanto para acceder a la formación PIR se debería exigir previamente el MUPGS. Ello dejaría la formación en diez años. Por el contrario el mundo universitario se muestra reticente, prefiriendo el diseño actual.

Las ventajas probablemente se deriven de que garantiza una muy completa formación en el PEPC y en que normalizaría y extendería el MUPGS como etapa de formación.

Los inconvenientes son precisamente derivados de lo anterior ya que tiende a convertir el MUPGS como el punto de referencia de TODA LA ACTIVIDAD PSICOLOGICA en el ámbito sanitario, lo que no refleja la realidad de la profesión de psicólogo/a. Obviamente ello redundaría en la viabilidad y rentabilidad del resto de másteres universitarios. Respecto al número de plazas o son aumentadas muy sensiblemente por la red pública, lo que no parece muy probable o se debería dar entrada a la formación de especialistas fuera de la red pública, lo que tampoco parece muy viable.

Tampoco es de despreciar el argumento de que si a ello se añade la especialización en aspectos concretos tal como la hemos defendido aquí, la formación se alargaría hasta los doce años. El tema está encima de la mesa. 


\section{A modo de resumen. Perspectivas de futuro}

La mayoría de temas en discusión sobre la regulación de la práctica de la PCS y sus circuitos de formación han sido planteados en las líneas anteriores, pero sirvan estas breves palabras de resumen. En nuestra opinión el tema más importante es la incorporación al programa PIR y por lo tanto al acceso al título de PEPC, de nuevas especialidades sanitarias psicológicas como la neuropsicología (ver p. 6.). De hecho ya se ha producido con el proyecto de constituir el PEPC infanto-juvenil, aunque ello es una especialidad basada en la especificidad de la población, no de la disciplina y, ello lo hace mucho más fácil. No es el caso aquí de iniciar una discusión sobre cómo debería ser esta incorporación de estas nuevas especialidades, pero llamamos la atención sobre el problema.

Una segunda cuestión importante es estudiar la posibilidad de incorporar a los PGS en la red pública. En nuestra opinión, ello es administrativamente posible y de hecho nos constan algunas experiencias en la red pública sanitaria catalana. Cuando pensamos en ello estamos pensando, cómo íntimamente relacionada, la cuestión de la introducción de los Psicólogos/as en la red pública de Atención Primaria y, finalmente vinculada con lo anterior está la cuestión de la relación entre los PEPC y los PGS, ya que nos parece obvio que tarde o temprano convivirán en la red de atención pública, luego habrá que establecer las reglas de relación profesional y jerárquica si la hay, entre las dos figuras.

Una tercera y última conclusión, es la necesidad de regulación legal de aquellos circuitos formativos que se encuentran en una situación alegal, como sería el itinerario formativo descrito en la posibilidad 3 (Grado - Formación Específica) en los que se podrían encontrar la neuropsicología o la psicoterapia.

\section{Referencias Bibliográficas}

Agencia Nacional de Evaluación de la Calidad y Acreditación(ANECA) (2005). Libro Blanco del Título del Grado en Psicología. Recuperado de: http://www.aneca.es/var/media/150356/libroblanco_psicologia_def.pdf

Defensor del Pueblo (2020). Recomendación sobre Atención psicológica en el Sistema Nacional de Salud. Recuperado de: https://www.defensordelpueblo.es/resoluciones/estudio-de-necesidades-e-incremento-de-laatencion-psicologica-en-el-sistema-nacional-de-salud/

Comisión Europea (2016). Mutual evaluation of regulated professions. Overview of the regulatory framework in the health services sector - psychologists and related professions. Recuperado de: https://ec.europa.eu/ docsroom/documents/16683/attachments/1/translations/en/renditions/native

España. Audiencia Nacional, Sala de lo Contencioso Administrativo, sección 6a . Sentencia 372/2016 de 07 de octubre. Recuperada de: http://www.poderjudicial.es/search/doAction?action=contentpdf\&databasematc h=AN\&reference=7846957\&links=\%22372\%2F2016\%22\&optimize=20161024\&publicinterface=true 